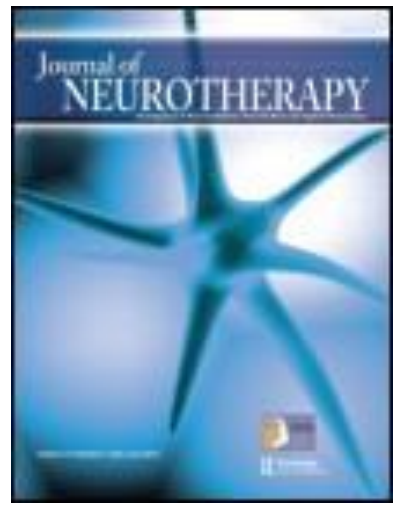

Journal of Neurotherapy: Investigations in Neuromodulation, Neurofeedback and Applied Neuroscience

\title{
Neurofeedback and Basic Learning Theory: Implications for Research and Practice
}

Leslie H. Sherlin $^{a b c}$, Martijn Arns ${ }^{\text {de }}$, Joel Lubar ${ }^{f}$, Hartmut Heinrich ${ }^{\text {gh }}$, Cynthia Kerson ${ }^{\text {ijk }}$, Ute Strehl '\& M. Barry Sterman m

a Neurotopia, Inc. , Los Angeles, California, USA

b Nova Tech EEG, Inc. , Mesa, Arizona, USA

C Southwest College of Naturopathic Medicine, Tempe, Arizona, USA

d Research Institute Brainclinics, Nijmegen, The Netherlands

e Department of Experimental Psychology, Utrecht University, Utrecht, The Netherlands

$\mathrm{f}$ University of Tennessee, Knoxville, Tennessee, USA

$\mathrm{g}$ Department of Child and Adolescent Mental Health , University of Erlangen-Nürnberg, Erlangen, Germany

h Heckscher-Klinikum, München, Germany

i ISNR Research Foundation, San Rafael, California, USA

j Brain Science International, Pleasanton, California, USA

k Marin Biofeedback, San Rafael, California, USA

I University of Tubingen, Tubingen, Germany

m University of California , Los Angeles, California, USA Published online: 30 Nov 2011.

To cite this article: Leslie H. Sherlin , Martijn Arns, Joel Lubar , Hartmut Heinrich , Cynthia Kerson, Ute Strehl \&

M. Barry Sterman (2011) Neurofeedback and Basic Learning Theory: Implications for Research and Practice, Journal of Neurotherapy: Investigations in Neuromodulation, Neurofeedback and Applied Neuroscience, 15:4, 292-304, DOI: 10.1080/10874208.2011.623089

To link to this article: http://dx.doi.org/10.1080/10874208.2011.623089

PLEASE SCROLL DOWN FOR ARTICLE

(C) International Society for Neurofeedback and Research (ISNR), all rights reserved. This article (the "Article") may be accessed online from ISNR at no charge. The Article may be viewed online, stored in electronic or physical form, or archived for research, teaching, and private study purposes. The Article may be archived in public libraries or university libraries at the direction of said public library or university library. Any other reproduction of the Article for redistribution, sale, resale, loan, sublicensing, systematic supply, or other distribution, including both physical and electronic reproduction for such purposes, is expressly forbidden. Preparing or reproducing derivative works of this article is expressly forbidden. ISNR makes no representation or warranty as to the accuracy or completeness of any content in the Article. From 1995 to 2013 the Journal of Neurotherapy was the official publication of ISNR (www. Isnr.org); on April 27, 2016 ISNR acquired the journal from Taylor \& Francis Group, LLC. In 2014, ISNR established its official open-access journal NeuroRegulation (ISSN: 2373-0587; www.neuroregulation.org).
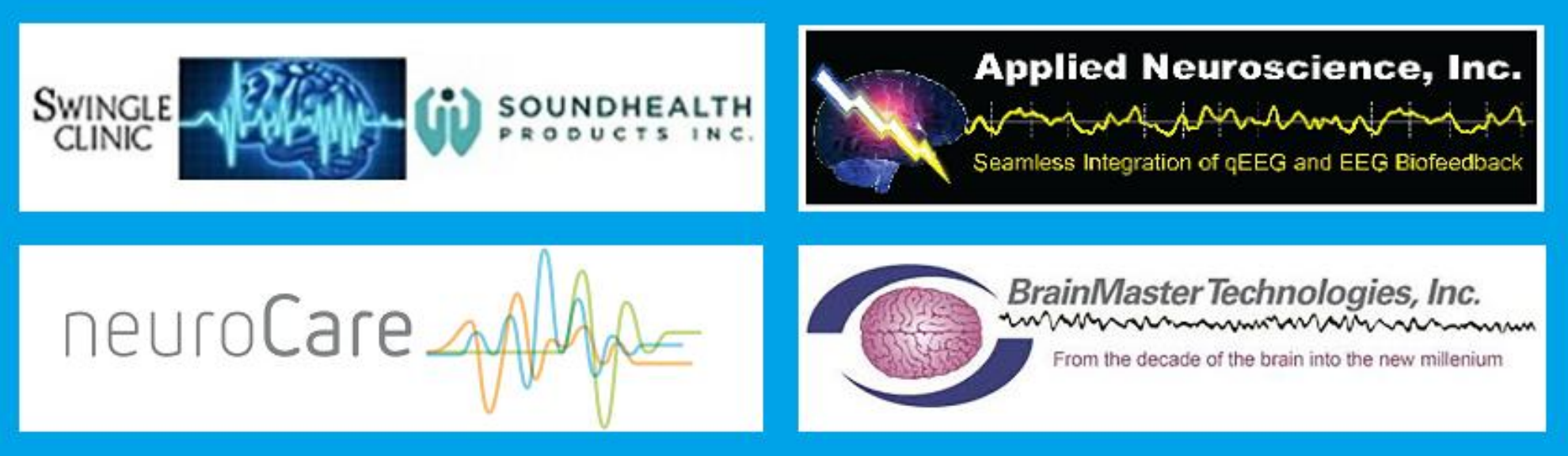


\title{
REVIEW ARTICLES \\ NEUROFEEDBACK AND BASIC LEARNING THEORY: IMPLICATIONS FOR RESEARCH AND PRACTICE
}

\author{
Leslie H. Sherlin ${ }^{1,2,3}$, Martijn Arns ${ }^{4,5}$, Joel Lubar ${ }^{6}$, Hartmut Heinrich ${ }^{7,8}$, Cynthia Kerson ${ }^{9,10,11}$, \\ Ute StrehI $^{12}$, M. Barry Sterman ${ }^{13}$ \\ ${ }^{1}$ Neurotopia, Inc., Los Angeles, California, USA \\ ${ }^{2}$ Nova Tech EEG, Inc., Mesa, Arizona, USA \\ ${ }^{3}$ Southwest College of Naturopathic Medicine, Tempe, Arizona, USA \\ ${ }^{4}$ Research Institute Brainclinics, Nijmegen, The Netherlands \\ ${ }^{5}$ Department of Experimental Psychology, Utrecht University, Utrecht, The Netherlands \\ ${ }^{6}$ University of Tennessee, Knoxville, Tennessee, USA \\ ${ }^{7}$ Department of Child and Adolescent Mental Health, University of Erlangen-Nürnberg, \\ Erlangen, Germany \\ ${ }^{8}$ Heckscher-Klinikum, München, Germany \\ ${ }^{9}$ ISNR Research Foundation, San Rafael, California, USA \\ ${ }^{10}$ Brain Science International, Pleasanton, California, USA \\ ${ }^{11}$ Marin Biofeedback, San Rafael, California, USA \\ ${ }^{12}$ University of Tubingen, Tubingen, Germany \\ ${ }^{13}$ University of California, Los Angeles, California, USA
}

Brain activity assessed by electroencephalography (EEG) has been demonstrated to respond to conditioning techniques. The concept of modulating this activity has been called EEG biofeedback, more recently neurofeedback, and is based on operant learning principles. Technological advancements have significantly enhanced the ease and affordability of recording and analyzing brain activity. Thus, properly trained practitioners can implement these conditioning strategies in their practice. Recent research indicating evidenced-based efficacy has made this technique a more viable option for clinical intervention. The objective of this article is to highlight the learning principles that have provided the fundamentals of this neuromodulatory approach. In addition, it is recommended that future applications in clinical work, research, and development adhere to these principles.

\section{INTRODUCTION}

Electroencephalographic (EEG) brain activity has been demonstrated to be responsive to operant and classical conditioning. This technique has been called EEG biofeedback and more recently neurofeedback. The developments of computer technologies have advanced, and now it is affordable and convenient for private setting providers to access software and hardware that can be used for conditioning the EEG. This article highlights the learning principles fundamental to the successful implementation of this neuromodulation approach. It is our contention that future applications in clinical work, research, and development should not stray from the already-demonstrated basic principles of learning theory until empirical evidence demonstrates otherwise. Considerations for

Received 12 August 2011; accepted 1 September 2011.

We acknowledge the contributions of research assistant Noel Larson, MA, for her involvement in researching the previous literature as well as her review and proofing of the manuscript.

Address correspondence to Leslie H. Sherlin, PhD, 8503 East Keats Avenue, Mesa, AZ 85209, USA. E-mail: lesliesherlin@mac.com 
the well-developed learning theory principles should be made in the practical implementation of neurofeedback.

\section{HISTORICAL PERSPECTIVE}

Ivan Pavlov established classical conditioning in 1927 in a series of experiments measuring the salivation of dogs in relation to the presentation of food. In short, unconditioned responses, originally called "inborn reflexes" (Pavlov, 1927), are reactions to stimuli that require no learning to occur. These unconditioned responses are often the result of naturally occurring stimuli and are useful in survival, such as salivating at the sight of food to increase digestion speed. In this example, food is labeled an unconditioned stimulus, or a stimulus that automatically triggers an unconditioned response (salivating; Pavlov, 1927). Pavlov soon discovered that a conditioned response, such as salivation when hearing the footsteps of the person bringing food to the dog, can be elicited by a conditioned stimulus, in this case the footsteps. He coined this process classical conditioning (Pavlov, 1927). However, classical conditioning does not explain all changes of behavior or the emergence of new behaviors. To explain the emergence of newly learned behaviors that were not a result of classical conditioning, Thorndike (1911/1999) conceptualized operant conditioning by proposing the "Law of Effect," which stated, responses that produce a satisfying effect in a particular situation become more likely to occur again in that situation, and responses that produce a discomforting effect become less likely to occur again in that situation.

Operant conditioning was most refined by the revered psychologist B.F. Skinner (1948). Through his work, the rules for operant conditioning were clearly defined. Operant conditioning can increase a preferred behavior and decrease an undesired behavior by providing a reward or punishment. A reward is any event (presentation of food, tones, etc.) that follows a specified response that is considered to be desirable and is intended to promote the specified response to occur again under the same conditions. A punishment, on the other hand, is any event that follows a specified response that is not desirable and is designed to prevent the specified response from occurring again. If the consequence of the reward or punishment increases or decreases the probability of the response, the response becomes reinforced. Therefore any event that follows the response to a stimulus (reward or punishment) and increases or decreases the likelihood of a behavior is considered a reinforcer. It is important to note that the terms reward and punishment do not mean the same as reinforcer. A reward or punishment specifically refers to the event following a response, whereas the reinforcer is an effective reward or punishment in increasing or decreasing the likelihood of the response occurring again respectively (i.e., strengthening of the response). We recognize that this sequence can be difficult to conceptualize. In chronological order, a stimulus occurs that is followed by a behavioral response. A reward or punishment is presented in reaction to the response in order to promote or suppress the response. If the reward or punishment achieves this goal, it is labeled a reinforcer.

The words positive and negative in operant conditioning terminology imply the presentation or removal of reinforcement. There are many ways these terms are combined that have effects on behavior. To illustrate the possible combinations, a few examples are provided. A child may be more likely to pay attention in class if stickers are presented afterward (positive reward). Conversely, a child might be more likely to pay attention if he is allowed to skip study hall time (negative reward). A child may be more likely to pay attention in class if she is given extra work to complete if she does not pay attention (positive punishment). Finally, a child may be more likely to pay attention if he is not allowed to participate in recreation time if he fails to pay attention (negative punishment). In general, the term positive is an additive process of reward or punishment, and the term negative is the removal of a reward or punishment to promote the desired behavior.

The timing of the reinforcement of a behavior is critical to learning because delays as small as a fraction of a second can decrease the 
strength of the conditioning (Skinner, 1958). The contingent relationship between the behavior and the reinforcement must be evident to the learner. Reinforcement schedules are primarily either continuous reinforcement (this describes a schedule where a response is reinforced rapidly each time it occurs) or intermittent reinforcement (i.e., not every occurrence of the response is rewarded). There are four types of intermittent reinforcement schedules:

1. Fixed-ratio reinforcement schedule, in which the subject must reproduce the correct response a set number of times before reinforcement is presented. A fixed-ratio schedule does not depend on the speed of the responses, only the number of responses.

2. Variable-ratio schedules require an unpredictable number of responses to attain reinforcement.

3. Fixed-interval schedules are similar to Fixedratio reinforcement schedules except that both the response must occur as well as a set interval of time.

4. Variable-interval schedules require the response and varied time segments to occur before the reinforcement is made available.

For neurofeedback, most current implementations and studies have utilized a continuous reinforcement schedule within sessions. To facilitate transfer of the self-regulation skill to everyday life, some protocols use transfer trials. Transfer trials are where no feedback is provided during the given period, only feedback on whether the trial was "correct" or "incorrect" (Rockstroh, Elbert, Birbaumer, \& Lutzenberger, 1982). This process refers to a response generalization, which is explained later. However, it is very important to neurofeedback that the final representation of a response can be reached by reinforcing successive approximations of the desired response, a process termed shaping (Skinner, 1958).

Shaping can be used to decrease learning time for the final response by breaking information down into small steps that are easier to accomplish, gradually requiring more steps before reinforcement occurs. Utilizing chaining, one can increase the complexity of the final response. Like many links in a chain, behavioral responses may be reinforced individually at first, but finally the whole sequence of events must occur before reinforcement. For example, an illustration of chaining is the pigeons that Skinner taught to "play" ping-pong (Skinner, 1962). The pigeons were taught behaviors successively, but each was dependent on the previous to occur. Such complex behavior can only be conditioned employing the principles of learning theory by chaining and reinforcing successive approximations of behavior.

Two other related concepts integral to learning theory are habituation and sensitization, which are both forms of nonassociative learning. Habituation is the learning process that results in an individual decreasing the response to a stimulus due to the reduction in reaction to novelty. This occurs due to repeated exposure and is typical when the individual is continuously exposed to the same stimuli (Groves \& Thompson, 1970). In more recent experimental studies of learning, it has been demonstrated that differential rates of habituation (e.g., to a novel open field) occur between animals of high and low general learning abilities. Because conditioning principles of neurofeedback have largely been applied in the clinical setting, there is a possibility that there is increased habituation and less behavior conditioning in individuals who have lower capacities for learning (Light, Grossman, Kolata, Wass, \& Matzel, 2011).

Sensitization, similar to habituation only through the repetitive nature of the stimuli, occurs when the individual has an amplification of a sensation or awareness of the particular stimulus. The individual may find what was a normal response to be painful or otherwise annoying and will attempt to avoid the stimulus even if it once was pleasing or an indication of a reward. Sensitization of the nervous system is influenced by behaviors that are increased or decreased by positive and negative reinforcement. The presence of clinical symptomatology may additionally amplify the presentation and impede improvements (Treisman \& Clark, 2011). This consideration should also be given 
when determining learning responses and as explanation for less-than-ideal conditioning responses. The neuronal basis of habituation and sensitization have been demonstrated in experiments with the sea slug Aplysia (Kandel \& Taut, 1965a, 1965b), demonstrating that these learning principles represent very basic forms of learning, even present in "simple" nervous systems. This work earned Kandel the Nobel Prize and is most recently described by Hawkins, Kandel, and Bailey (2006).

\section{CLASSICAL CONDITIONING OF BRAIN ACTIVITY}

The first demonstrations that the brain activity, more specifically, the alpha blocking response, could be classically conditioned was reported almost simultaneously in France by Durup and Fessard (1935) and in the United States by Loomis, Harvey, and Hobart (1936). Loomis et al. described that in a completely dark room pairing a low auditory tone with a light stimulus resulted in the auditory stimulus becoming the conditioned stimulus leading to the conditioned response - in this case, blocking of the alpha. They also observed that extinction took place if the low tone was presented several times in absence of the light stimulus.

The first systematic studies investigating classical conditioning of the alpha-blocking response were conducted several years later. Jasper and Shagas (1941a) demonstrated that the simple conditioned response of alpha blocking was equal or longer with the conditioned stimulus as it was by the unconditioned stimulus after only 10 paired presentations (Jasper \& Shagas, 1941a). In addition, they demonstrated that alpha-blocking conditioned response could be conditioned with many different styles of reinforcement (cyclic, delayed, trace, differential, differential delayed, and backward) and hence demonstrated that conditioning of the alpha-blocking response fulfilled all of the Pavlovian types of conditioned responses.

In the same year, Knott and Henry (1941) were skeptical about the conditioning of the alpha block and conducted their own research to differentiate if conditioning or sensitization was operating in the previous studies. They demonstrated that the connection between the conditioned stimulus and the alpha block occurred less frequently with more and more trials. They believed it was an anticipatory alpha block response that was being conditioned. Thus, they tentatively affirmed that conditioning was occurring and discussed the implications of it, noting that the operant conditioning of the EEG is a different process making it "possible to postulate an association between a cortical state following a peripheral stimulus (conditioned stimulus) and the cortical state preceding the unconditioned response" (p. 142). They elaborated that this theory would work in conjunction with the-then predominant - motor theories of psychology.

Also in 1941, Jasper and Shagas collaborated to build upon the Pavlovian conditioning of the occipital alpha blocking. From previous research, they summarized that it is clear that alpha blocking is not simply an unconditioned response to light. It may also become a conditioned response of stimuli beyond light alone. In their next study, Jasper and Shagas (1941b) were successful in demonstrating the operant conditioning of the alpha block by having subjects voluntarily say subvocally "block" and press a button followed by a subvocal "stop" and release of the button. The button switched on the light, serving as the unconditioned response, associating the subvocal command with alpha blocking and hence becoming the conditioned response. This was actually the first demonstration of "voluntary control" of the EEG in humans.

In 1963, Sterman, and later in the same year Clemente, Sterman, and Wyrwicka, took this classical conditioning of brain activity one step further. They demonstrated in cats that the EEG synchronization and behavioral manifestations of sleep could be conditioned with a tone (conditioned stimulus) and basal forebrain stimulation (unconditioned stimulus), where the conditioned stimulus eventually resulted in sleep preparatory behavior. Furthermore, they also reported that generalization for different tone frequencies and discrimination of the conditioned response had occurred, suggesting that conditioning principles could be applied. 
Milstein (1965) summarized the controversy of conditioning EEG responses, noting that initially it seemed clear that alpha blocking was always the result of the presentation of light (Bagchi, 1937) and that this unconditioned response could be conditioned to neutral stimuli (Gastaut et al., 1957; Jasper \& Shagass, 1941b; Jus \& Jus, 1957; Knott \& Henry, 1941; Loomis et al., 1936; F. Morrell \& Ross, 1953; Travis \& Egan, 1938). However, Milstein (1965) then pointed to the number of studies that failed to show consistency across all individuals in the alpha-blocking response (Redlich, Callahan, \& Mendelson, 1946) and the variability in the response duration (Jus \& Jus, 1960; L. Morrell \& Morrell, 1962) or even reduction in the response all together (Wells, 1963).

With all of this in mind, Milstein designed a study to determine if a neutral stimulus could produce alpha blocking without being paired with a stimulus that generally elicits the response. Conversely, Milstein also set out to test the following: If the conditioned stimulus was paired with the unconditioned stimulus during nonalpha EEG activity, could the conditioned stimulus then elicit the alpha-blocking response during alpha activity trials? In Milstein's experiment there were 11 different conditions to answer these questions, including habituation periods, test periods, and rest periods, and all were followed by a classical conditioning period. The results indicate that alpha blocking could be conditioned. However, because it was not necessary to pair the conditioned stimulus with the unconditioned stimulus, it is possible that Knott and Henry's (1941) idea that instead of true conditioning the subjects simply became sensitized to the tone seemed plausible. Therefore, it still has not been determined if the results just summarized should be considered classical conditioning or simply reflect sensitization. However, the results do demonstrate that the EEG is subject to learning principles.

\section{OPERANT CONDITIONING OF BRAIN ACTIVITY}

In 1962, the first results of voluntary control over alpha activity based on operant conditioning principles were presented by Kamiya (2011). Several years after Kamiya's reports, Sterman's laboratory demonstrated operant conditioning of sensory motor rhythm (SMR) activity in the cat (Wyrwicka \& Sterman, 1968), which was demonstrated to have anticonvulsant properties (also see Sterman, LoPresti, \& Fairchild, 1969, 2011). This study initiated the beginning of clinical applications of operant conditioning of the EEG.

Given some conflicting results in the early years on the operant conditioning of alpha activity, Hardt and Kamiya (1976) suggested that there were methodological differences between the positive and negative outcomes. Specifically they reported that those who were using integrated amplitude to quantify the alpha response were affirming the conditioning process, whereas those who were using percent time were failing to support the conditioning response. Hardt and Kamiya pointed to the binary classification used in the percent-time system, above or below the threshold, which clearly ignores considerable information about the actual strength of the alpha signal. Collapsing a continuous variable, like the wide range of amplitude values in the alpha signal, into a binary or dichotomized variable always results in the loss of information about the "raw" or "true" variable (Tabachnick \& Fidell, 2007). This means that if the amplitude of alpha were to rise at the presentation of the conditioned stimulus but that increase falls just short of the arbitrary threshold used for percent-time categorization, then that response cannot be observed and it will appear as if conditioning has not occurred. Furthermore, Hardt and Kamiya (1976) pointed out that if reinforcement is withheld for those individuals who fall just short of the threshold, they will more than likely abandon "successful strategies" that result in alpha increases. On the other hand, the amplitude integration method displays the continuous information and is more sensitive to small changes in alpha amplitude, an important element for determining if conditioning of EEG responses can occur. Two aspects of the results prompt Hardt and Kamiya (1976) to say that "together [these results] suggest that 
the percent-time measure can be likened not only to a ruler with unequally spaced graduations, but worse, to a rubber ruler with unequal graduations which requires different degrees of stretch to account for different threshold settings" (p. 72).

Lansky, Bohdaneck, Indra, and Radii-Weiss (1979) attempted to counter Hardt and Kamiya (1976) regarding percent-time alpha feedback versus amplitude-integration. They started by pointing out that the percent time is a continuous variable because it ranges from 0 to $100 \%$ (but fail to appreciate the importance of the individual being able to see small changes during training). Lansky et al. then noted that in longer training periods, say over minutes, amplitude-integral method does not allow for quick display of alpha spindles, unlike the percent-time ratio. They also challenge the ideas that (a) an integral method could fit into a Poisson distribution because it is discrete and (b) the rectangle probability distribution of the percent-time method that was reported by Hardt and Kamiya. The rectangle probability distribution was not confirmed with any statistical test and is supposedly discrepant with the empirical findings reported by Lansky et al. Last, it is pointed out that both the spindle length and the power (or amplitude) of the alpha frequency are important aspects of future research, because they most likely have some relationship to each other and offer unique information about the EEG response. Furthermore, as of yet, neither method has been shown to be better than the other.

In 1964, Clemente, Sterman, and Wyrwicka reported an alphalike EEG synchronization in the parieto-occipital cortex, visible just after the animal was reinforced, which they named the post-reinforcement synchronization (PRS). Poschel and Ho (1972) demonstrated that this activity critically depends on the operant response, because providing the reinforcement alone without the requirement for an operant response (i.e., lever press) gradually weakensor habituates - the well-developed PRS. This phenomenon has also been observed in monkeys (Saito, Yamamoto, Iwai, \& Nakahama, 1973) and humans (Hallschmid, Mölle, Fischer, \& Born, 2002). In an attempt to understand the implications of operant conditioning of behavior and brain activity on learning, Marczynski, Harris, and Livezey (1981) hypothesized that the PRS of EEG that occurs in animals after the consumption of an expected food reward would be correlated to a greater ability to learn. This hypothesis was derived from the knowledge that the PRS occurs not only "in the primary and secondary visual cortex but also over the association cortex (suprasylvian gyri) of both hemispheres" (Marczynski et al., 1981 , p. 214). After successfully training 25 of 27 cats to press a lever to receive $1 \mathrm{ml}$ of milk, the authors were able to demonstrate a significant positive correlation between greater PRS indices and faster learning.

Cognition-impairing drugs like scopolamine and atropine block the PRS (Marczynski, 1971), further supporting the role of PRS in consolidation of information processing. Hallschmid et al. (2002) investigated the PRS further in humans and found that a lower-alpha synchronization in humans indicates "distinct similarities to the PRS phenomena observed in animals under comparable conditions" (p. 214). It is suggested that there is a correlation between the magnitude of the PRS and learning abilities in cats (Marczynski et al., 1981). Likewise, in humans, functional relations between lower alpha and theta activity during learning processes have been suggested (Doppelmayr, Klimesch, Pachinger, \& Ripper, 1998; Klimesch, 1999), thus further confirming the role of PRS in learning.

\section{IMPLICATIONS FOR PRACTICE}

Based on the established learning theoretical principles just outlined, there are techniques that are crucial elements when designing a neurofeedback study and are important aspects to be implemented in practice. Next we discuss the previously mentioned learning theory concepts and the specific implication for research and practice.

\section{Speed of Reinforcement}

It is well known that different EEG filters possess different properties. The most important aspect 
is that the more specific a filter is (often referred to as a higher order filter), the more data points that are required to make such a calculation. This results in a longer delay of the feedback signal. As was pointed out by Skinner (1958), the timing of the reinforcement or punishment of a behavior is critical to learning, as a delay as small as a fraction of a second can decrease the strength of the conditioning. Therefore, it is important to understand the specific filter settings used and set them appropriately. In general, the faster the response time of the filter, the better. There is no fixed rule on what is the minimum or maximum acceptable delay of a filter, and this also depends on the "required specificity," but based on Felsinger and Gladstone (1947) and Grice (1948), the latency should not exceed 250 to $350 \mathrm{~ms}$ (see Figure 1, from Grice, 1948). Practically speaking, this is equivalent to an FIR filter with a fifth-order Butterworth filter.

\section{Type of Reinforcement}

As was presented earlier, the concept of PRS is a reflection of reinforcement, and the amount of PRS is clearly associated with the speed of learning (Hallschmid et al., 2002; Marczynski et al., 1981). Therefore, for optimal learning to take place it is important that the "feedback" is designed in such a way that a PRS can occur.

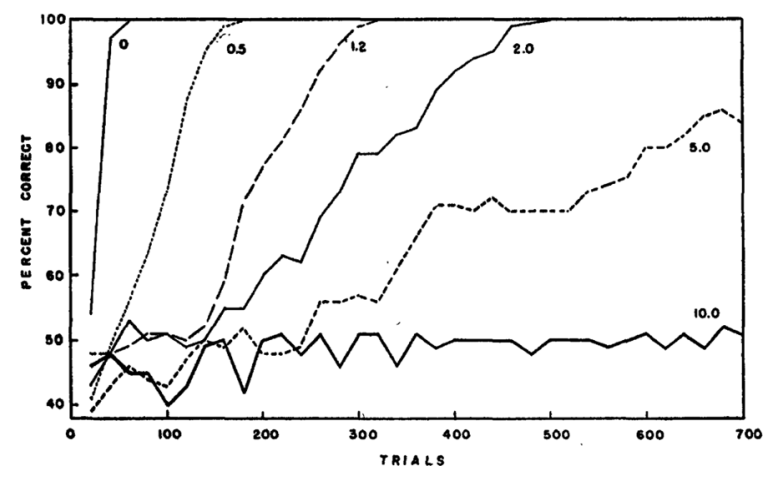

FIGURE 1. The number of trials required for response acquisition as a function of the delay of reinforcement. Note. This figure clearly demonstrates that the faster the reinforcement is provided the faster the learning. From "The Relation of Secondary Reinforcement to Delayed Reward in Visual Discrimination Learning," by G. R. Grice, 1948, Journal of Experimental Psychology, 38, pp. 1-16. Copyright 1948 by the American Psychological Association. Reprinted with permission.
This requires a discrete feedback setup most closely resembling the early neurofeedback equipment from 1970s that had discrete Butterworth 4 pole filters for specific frequency bands described by Sterman, Macdonald, and Stone (1974). When all criteria were met for $.5 \mathrm{~s}$, there was a green light and a "ding" sound, resulting in a discrete reward allowing for a PRS to take place.

Consequently, complex games offered in some products are contraindicated, given that the level of continuous feedback does not allow for a PRS complex to occur because it is too difficult for the learner to extract meaningful information. Operant learning involves the formation of a response-reinforcer association. Complex games are much more likely to "overshadow" the response-reinforcer association by the formation of a more salient stimulusreinforcer association (Pearce \& Hall, 1978). This practically means they will associate the reinforcement with the stimulus rather than the desired specific brain behavior response. In addition, the presentation of a signal during the interval between the response and the reinforcement can block learning (Williams, 1999). Therefore, in the application of neurofeedback, one "should stress exercise rather than entertainment" (Egner \& Sterman, 2006). The reinforcement should lead to "knowledge of results." Therefore, it should specifically inform the learner whether the response was right or wrong and to what extent the brain signal changed. To date, no studies could be found empirically testing the efficacy of continuous feedback, such as a game or video presentation, to applications with a discrete feedback of game or video presentations. However, the theory and evidence previously described provides sufficient indication that the discrete feedback would achieve superior results.

\section{Shaping}

Another aspect of learning theory entails the "shaping procedure" of operant conditioning. During operant conditioning of a behavior, or in this case, brain activity, shaping occurs by adjusting thresholds in an a priori direction to promote learning. Based on the earlier work 
of Hardt and Kamiya (1976), the feedback parameters and reward should not utilize automatic calculation of thresholds. The continuous updating of a threshold is a "moving target" and the reward feedback can be provided to the learner for a given percentage even when the desired activity is changing in the opposite direction. In auto-thresholding, the reward feedback will be given for moving in the desired direction in a momentary response. This may not actually increase or decrease the amplitude in the desired training direction in consideration of the baseline level. Hence, auto-thresholding techniques do cause differential effects, which can also be understood from learning theory.

This can be illustrated by a practical example of a screaming child. The goal is to teach the child to no longer scream, and we "punish" the child every time it screams. In an auto-thresholding model eventually the child will be punished for "raising its voice," that is, auto-thresholding is blind to the quality of the behavior being trained. In addition to this point, if the learner begins to fatigue, lose interest, or even stop actively participating in the training, the reward signals continue to be provided irrespective of whether they are producing the desired behavior. They are, in fact, being rewarded for only changing the behavior based on the previous averaged time period, which may not be an actual change from the starting behavior point. Even worse, it may actually be in the opposite direction than the desired training parameter. Consider if the learner is being asked to reduce the amplitude of a given frequency band and the threshold is calculated automatically, they will always be getting a percentage of feedback even if the amplitudes are rising across time. If this occurs, at best, the learner may show improvements only if they continually demonstrate change in the desired direction. It is possible that they may show no learning and no effect. Finally, at worst they could effectively train in the opposite direction and result in an increase in aberrant and negative behaviors. Again, although there have been no studies directly comparing an auto-thresholding procedure to a non-auto-thresholding procedure in neurofeedback, there is a considerable body of theoretical and empirical evidence supporting the non-auto-thresholding procedure for operant conditioning.

Whether shaping is needed at all depends on if the ultimate desired behavior can be operationalized. For example, in paradigms such as slow cortical potentials (SCP) feedback there is not such a known goal. It does not matter whether the shift of the SCP deviates about 2 or $7 \mu \mathrm{V}$ from baseline. There is no "norm" to be reached by a learner. Only the positive or negative deviation from baseline is important. If at all, shaping procedures are used here for "comorbid" behaviors as not being able to remain seated, not to produce artifacts, and so on. Therefore, knowledge about the brain activity to be trained is also crucial in applying the right learning theoretical principles, as is further outlined in the upcoming Specificity section.

Finally as described earlier, the autothresholding procedure precludes the possibility of the neurofeedback being applied in a "blinded" fashion. When investigating in a double-blind fashion, one has to resort to techniques like "auto-thresholding" to force the blinded methodology impractically upon the neurofeedback intervention. This will contribute to null findings, as one of the primary components of the neurofeedback training is based in the learning principle of shaping that will completely violated in the auto-thresholding procedure.

\section{Specificity}

Philippens and Vanwersch (2010) wanted to address what they perceived to be a lack of well-controlled, scientific investigations of the efficacy of neurofeedback. As a result, they designed a laboratory study utilizing telemetric neurofeedback with Marmoset monkeys $(N=4)$ to determine if, through operant conditioning, these monkeys could increase the SMR. They rewarded the presence of "high alpha," and they clarified this as a peak frequency between 11 to $14 \mathrm{~Hz}$. All four monkeys were able to significantly increase the presence of this frequency, though each monkey had a 
unique learning curve. One monkey learned to increase this frequency so well that by Session 3 , all 35 possible rewards were obtained in the last two of the six 5-min epochs. Due to this fast learning, this monkey was exposed to extinction training, where the recording was done without the possibility of rewards. In that extinction trial, Philippens and Vanwersch reported that this monkey clearly expressed expectant behavior after successful EEG changes, which they cite as evidence that the monkey learned to increase high alpha to obtain the reward. They reported that the short training time necessary for this level of success was possible related to the intracortical electrodes because this significantly decreases the noise measured by the scalp electrodes. This demonstrated that the better the specificity of the signal or behavior trained, the faster the learning takes place, in agreement with what would be expected from learning theory.

For operant conditioning it is very important to be aware of specifically "what behavior" is being conditioned in order to achieve learning and to improve the specificity. In the case of neurofeedback, knowledge about the trained brain rhythms, and knowledge about the neurophysiology of the EEG, is crucial. For example, when performing SMR neurofeedback, it is important to be aware of how SMR occurs, namely, in spindles with a specific duration. Therefore, better results are obtained when a specific SMR spindle with duration of $0.25 \mathrm{~s}$ is reinforced, rather then reinforcing any excursion above the SMR amplitude threshold. By including metrics such as "time above threshold" or "sustained reward period," the feedback will become more specific to the true SMR rhythm. The same concept was illustrated in the example of Hardt and Kamiya (1976) regarding alpha neurofeedback. As was previously described, the same applies to SCP neurofeedback, where specifically the polarity of the EEG is reinforced (i.e., negativity vs. positivity), not requiring shaping but simply a response in the right direction.

\section{Artifacts}

Muscle activity, eye movements, electrocardiogram, breathing, and other environmental and physiological factors may produce artifacts. Whether this is intentional or not, as soon as such activity is not detected as an artifact and is rewarded, an incorrect learning process will take place, such as rewarding eye blinks. Conversely, it is easy for the learner to reduce frontal delta across time simply by learning to behaviorally produce fewer eye blinks. Therefore, equipment should be able to detect artifacts online, or better in real time. In addition, the therapist or investigator has to closely observe the learner to avoid this "artifact-driven" feedback, thus also improving the specificity of what is trained.

\section{Secondary Reinforcement}

As we have seen from the study by Clemente et al. (1964), a reward needs to be not only discrete but also rewarding given that PRS was most clearly present when the reward was milk as opposed to water. So how can we make the feedback employed more interesting for the learner? For some clinics, monetary rewards or prizes are used as a secondary reinforcement. For example, if a child gains a specific number of points, he or she obtains a prize. It is very important that any monetary or other form of secondary reinforcer be tied to the learning process and not for simple participation (e.g., showing up for appointments without producing any change in EEG). Several animal studies have demonstrated a facilitation of learning by using a secondary reinforcer (e.g., Grice, 1948). However, this has not been investigated for the longer training periods used in clinical practice (after a session of $20 \mathrm{~min}$ ), so at this moment this is only a clinical observation.

\section{Generalization}

When a client is performing neurofeedback in the clinic, he or she will eventually learn to exercise control over the trained parameter. This skill is - to some degree-associated with the environment of the clinic and the relationship with the practitioner and may not be used outside this setting. To also have the benefits of this self-regulation skill in daily life, a process called generalization should occur, so the learned control is also exercised outside the clinic and without the feedback. In most SCP 
studies and many clinical settings, this has been facilitated by also including transfer trials, which are neurofeedback trials without the real-time feedback. Instead, the transfer trial provides information only at the end of the trial, whether it was successful or not (e.g., Drechsler et al., 2007). In the clinical setting, the client can be asked to rate the trial before knowing the score. This approach is further supported by studies on motor learning where better performance was observed as compared to a condition where feedback was provided $100 \%$ of the time (Winstein \& Schmidt, 1990). Furthermore, in SCP studies patients are provided with a card, which is a printout of the "success" screen. It serves as a cue (discriminative stimulus) to elicit the adequate brain state. The critical situations where and when self-regulation is most important (ADHD: before school tests/ Epilepsy: in seizure eliciting situations) are assessed during the treatment and practiced either in real-life situations or in role-play.

Of interest, several studies have demonstrated that generalization, both across time and across state, does take place. For example, Gani, Birbaumer, and Strehl (2008) demonstrated that the skill to regulate the SCP was still preserved at a 2 years' time follow-up. Recently, it was also demonstrated in a monkey that voluntarily modulated neural activity could indeed produce specific changes in cognitive function (Schafer \& Moore, 2011) suggesting a generalization from trained EEG parameters to behavior. Finally, Sterman, Howe, and Macdonald (1970) demonstrated that (12$15 \mathrm{~Hz})$ SMR training during wakefulness also resulted in the facilitation of $(12-15 \mathrm{~Hz})$ sleep spindles during sleep and a reduction of brief arousal disruptions during sleep, a finding that has recently been demonstrated in humans as well (Hoedlmoser et al., 2008). These last two studies also specifically demonstrate that successful operant conditioning has taken place.

\section{CONCLUSION}

The authors have concerns about many of the recent research studies that proclaim to have studied neurofeedback, many of which show poor results but also fail to present any indication of a learning process being administered and/or taking place. These results are being reported as neurofeedback studies. However, it may well be that because poorly designed control conditions, inappropriate protocols, inappropriate designs, or inappropriate equipment were used, in fact these studies have not investigated neurofeedback, defined as a learning process utilizing operant learning mechanisms of brain activity. This concern is based on the understanding of the nearly ninety years of research into conditioning, of both humans and animals, summarized in this article. Thus, we hope that future neurofeedback studies and clinical applications will be designed utilizing these basic principles of learning theory. If deviations from the established techniques are desirable for some reason, they should be empirically evaluated compared to those already demonstrating efficacy prior to implementation in private settings. We strongly encourage the practitioner to apply these same basic concepts and knowledge while fully understanding the requirements of an adequate setup of equipment as well as the training parameters and implementation.

It is additionally our hope that these wellestablished and empirically tested learning theory concepts be considered when evaluating outcome studies, being sure to not dismiss effective interventions based on studies that do not adhere to these concepts.

\section{REFERENCES}

Bagchi, B. K. (1937). The adaptation and variability of response of the human brain rhythm. Journal of Psychology, 3, 355-384.

Clemente, C. D., Sterman, M. B., \& Wyrwicka, W. (1963). Forebrain inhibitory mechanisms: Conditioning of basal forebrain induced EEG synchronization and sleep. Experimental Neurology, 7, 404-417.

Clemente, C. D., Sterman, M. B., \& Wyrwicka, W. (1964). Post-reinforcement EEG synchronization during alimentary behavior. Electroencephalography Clinical Neurophysiology, 16, 355-365. 
Cortoos, A., De Valck, E., Arns, M., Breteler, M. H., \& Cluydts, R. (2010). An exploratory study on the effects of tele-neurofeedback and telebiofeedback on objective and subjective sleep in patients with primary insomnia. Applied Psychophysiology and Biofeedback, 35, 125134. doi:10.1007/s10484-009-9116-z

Doppelmayr, M. M., Klimesch, W., Pachinger, T., \& Ripper, B. (1998). The functional significance of absolute power with respect to event-related desynchronization. Brain Topography, 11, 133-140.

Drechsler, R., Straub, M., Doehnert, M., Heinrich, H., Steinhausen, H. C., \& Brandeis, D. (2007). Controlled evaluation of a neurofeedback training of slow cortical potentials in children with attention deficit/hyperactivity disorder (ADHD). Behavioral and Brain Function, 3(1), 35.

Durup, G., \& Fessard, A. (1935). I. L'électrencéphalogramme de I'homme. Observations psycho-physiologiques relatives à l'action des stimuli visuels et auditifs [Electroencephalography human. Psycho-physiological observations relative to the action of visual and auditory stimuli]. L'année Psychologique, 36(1), 1-32. doi:10.3406/psy.1935.30643

Egner, T., \& Sterman, M. B. (2006). Neurofeedback treatment of epilepsy: From basic rationale to practical application. Expert Review of Neurotherapeutics, 6, 247-257. doi:10.1586/14737175.6.2.247

Felsinger, J. M., \& Gladstone, A. I. (1947). Reaction latency (StR) as a function of the number of reinforcements $(\mathrm{N})$. Journal of Experimental Psychology, 37, 214-228.

Gani, C., Birbaumer, N., \& Strehl, U. (2008). Long-term effects after feedback of slow cortical potentials and of theta-beta-amplitudes in children with attention deficit/hyperactivity disorder (ADHD). International Journal of Bioelectromagnetism, 10, 209-232.

Gastaut, H., Jus, C., Morrell, F., Storm Van Leeuwen, W., Dongier, S., Naquet, R., ... Were, J. (1957). Etude topographique des reactions electroencephalographiques conditionnees chez l'homme [Study of topographic EEG reactions condition in humans]. EEG and Clinical Neurophysiology, 9, 1-34.
Grice, G. R. (1948). The relation of secondary reinforcement to delayed reward in visual discrimination learning. Journal of Experimental Psychology, 38(1), 1-16.

Groves, P. M., \& Thompson, R. F. (1970). Habituation: A dual-process theory. Psychological Review, 77, 419.

Hallschmid, M., Mölle, M., Fischer, S., \& Born, J. (2002). EEG synchronization upon reward in man. Clinical Neurophysiology, 113, 1059-1065.

Hardt, J. V., \& Kamiya, J. (1976). Conflicting results in EEG alpha feedback studies: Why amplitude integration should replace percent time. Biofeedback and Self-Regulation, 1(1), 63-75.

Hawkins, R. D., Kandel, E. R., \& Bailey, C. H. (2006). Molecular mechanisms of memory storage in aplysia. Biology Bulletin Virtual Symposium on Marine Invertebrate Models of Learning and Memory, 210, 174-191.

Hoedlmoser, K., Pecherstorfer, T., Gruber, G., Anderer, P., Doppelmayr, M., Klimesch, W., \& Schabus, M. (2008). Instrumental conditioning of human sensorimotor rhythm (12-15 hz) and its impact on sleep as well as declarative learning. Sleep, 31, 1401-1408.

Jasper, H., \& Shagass, C. (1941a). Conditioning the occipital alpha rhythm in man. Journal of Experimental Psychology, 28, 373-387.

Jasper, H., \& Shagass, C. (1941b). Conscious time judgments related to conditioned time intervals and voluntary control of the alpha rhythm. Journal of Experimental Psychology, 28, 503-508.

Jus, A., \& Jus, C. (1957). L'application des réactions EEG conditionnées en neuropsychiatrie [The application of reactions are conditioned EEG in neuropsychiatry]. Electroenceph. clin. Neurophysiol, (Suppl. 7), 344-370.

Jus, A., \& Jus, C. (1960). Etude electro-clinique des alterations de conscience dansle petit mal [Study of electro-clinical alterations of consciousness in petit mal]. Studii si cercetari de Neurol, 5, 243-254.

Kamiya, J. (2011). The first communications about operant conditioning of the EEG. Journal of Neurotherapy, 15(1), 65-73. 
Kandel, E. R., \& Taut, L. (1965a). Heterosynaptic facilitation in neurons of the abdominal ganglion of aplysia depilans. Journal of Physiology, 181, 1-27.

Kandel, E. R., \& Taut, L. (1965b). Mechanism of heterosynaptic facilitation in the giant cell of the abdominal ganglion of aplysia depilans. Journal of Physiology, 181, 28-47.

Klimesch, W. (1999). EEG alpha and theta oscillations reflect cognitive and memory performance: A review and analysis. Brain Research Brain Research Reviews, 29(2-3), 169-95.

Knott, J. R., \& Henry, C. E. (1941). The conditioning of the blocking of the alpha rhythm of the human electroencephalogram. Experimental Psychology, 28, 134-144.

Lansky, P., Bohdaneck, Z., Indra, M., \& RadiiWeiss, T. (1979). Alpha detection: Some comments on Hardt and Kamiya, "Conflicting results in EEG alpha feedback studies." Biofeedback and Self-Regulation, 4, 127-131.

Light, K. R., Grossman, H., Kolata, S., Wass, C., \& Matzel, L. D. (2011). General learning ability regulates exploration through its influence on rate of habituation. Behavioural Brain Research, 223, 297-309.

Loomis, A. L., Harvey, E. N., \& Hobart, G. (1936). Electrical potentials of the human brain. Journal of Experimental Psychology, 19, 249.

Lubar, J. F. (2003). Neurofeedback for the management of attention deficit disorders. Biofeedback: A Practitioner's Guide, pp. 409-437.

Marczynski, T. J. (1971). Cholinergic mechanism determines the occurrence of reward contingent positive variation (RCPV) in cat. Brain Research, 28(1), 71-83.

Marczynski, T. J., Harris, C. M., \& Livezey, G. T. (1981). The magnitude of post-reinforcement EEG synchronization (PRS) in cats reflects learning ability. Brain Research, 204, 214-219.

Milstein, V. (1965). Contingent alpha blocking: Conditioning or sensitization? Electroencephalography and Clinical Neurophysiology, 18, 272-277.

Morrell, F., \& Ross, M. (1953). Central inhibition in cortical conditioned reflexes. A. M. A. Archives of Neurology Psychiatry, 70, 611-616.
Morrell, L., \& Morrell, F. (1962). Non-random oscillation in the response-duration curve of electrographic activation. Electroencephalography and Clinical Neurophysiology, 14, 724730. doi:10.1016/0013-4694(62)90086-X

Pavlov, I. P. (1927). Conditioned reflexes: An investigation of the physiological activity of the cerebral cortex (G. V. Anrep, Trans. \& Ed.). London, UK: Oxford University Press.

Pearce, J. M., \& Hall, G. (1978). Overshadowing the instrumental conditioning of a leverpress response by a more valid predictor of the reinforcer. Journal of Experimental Psychology: Animal Behavior Processes, 4, 356.

Philippens, I. H. C. H. M., \& Vanwersch, R. A. P. (2010). Neurofeedback training on sensorimotor rhythm in marmoset monkeys. NeuroReport, 21, 328-332.

Poschel, B. P., \& Ho, P. M. (1972). Postreinforcement EEG synchronization depends on the operant response. Electroencephalography and Clinical Neurophysiology, 32, 563-567.

Redlich, F. C., Callahan, A., \& Mendelson, R. H. (1946). Electroencephalographic changes after eye opening and visual stimulation. Yale Journal of Biology and Medicine, 18, 367-376.

Rockstroh, B., Elbert, T., Birbaumer, N., \& Lutzenberger, W. (1982). Slow brain potentials and behavior. Baltimore, MD: Urban \& Schwarzenberg.

Saito, H., Yamamoto, M., Iwai, E., \& Nakahama, H. (1973). Behavioral and electrophysiological correlates during flashfrequency discrimination learning in monkeys. Electroencephalography and Clinical Neurophysiology, 34, 449-460.

Schafer, R. J., \& Moore, T. (2011). Selective attention from voluntary control of neurons in prefrontal cortex. Science (New York, N. Y.), 332, 1568-1571. doi:10.1126/science.1199892

Skinner, B. F. (1948). "Superstition" in pigeons. Journal of Experimental Psychology, 38, 168-172.

Skinner, B. F. (1958). Reinforcement today. American Psychologist, 13, 94-99.

Skinner, B. F. (1962). Two "synthetic social relations." Journal of Experimental Analysis of Behavior, 5, 531-533. 
Sterman, M. B. (1963). Brain mechanisms in sleep, Chapter 4, Conditioning of induced EEG and behavioral sleep patterns: Doctoral Dissertation, University of California at Los Angeles, 80-99.

Sterman, M. B., Howe, R. C., \& Macdonald, L. R. (1970). Facilitation of spindle-burst sleep by conditioning of electroencephalographic activity while awake. Science, 167, 11461148.

Sterman, M. B., LoPresti, R. W., \& Fairchild, M. D. (1969). Electroencephalographic and behavioral studies of monomethylhydrazine toxicity in the cat. Alexandria, VA: Storming Media.

Sterman, M. B., LoPresti, R. W., \& Fairchild, M. D. (2010). Electroencephalographic and behavioral studies of monomethylhydrazine toxicity in the cat. Journal of Neurotherapy, 14, 293-300.

Sterman, M. B., Macdonald, L. R., \& Stone, R. K. (1974). Biofeedback training of the sensorimotor EEG rhythm in man: Effects on epilepsy. Epilepsia, 15, 395-417.

Tabachnick, B. G., \& Fidell, L. S. (2007). Using multivariate statistics (5th ed.). Boston, MA: Allyn and Bacon.
Thorndike, E. L. (1999). Animal intelligence (p. v). Bristol, UK: Thoemmes. (Original work published 1911)

Travis, L. E., \& Egan, J. P. (1938). Conditioning of the electrical response of the cortex. Journal of Experimental Psychology, 22, 524-531. doi:10.1037/h0056243

Treisman, G. J., \& Clark, M. R. (2011). A behaviorist perspective. Advances in Psychosomatic Medicine, 30, 8-21.

Wells, C. (1963). Electroencephalographic correlates of conditioned responses. In G. H. Glaser (Ed.), EEG and behavior (pp. 60-108). New York, NY: Basic Books.

Williams, B. A. (1999). Associative competition in operant conditioning: Blocking the response-reinforcer association. Psychonomic Bulletin \& Review, 6, 618-623.

Winstein, C. J., \& Schmidt, R. A. (1990). Reduced frequency of knowledge of results enhances motor skill learning. Journal of Experimental Psychology: Learning, Memory, and Cognition, 16, 677-691.

Wyrwicka, W., \& Sterman, M. B. (1968). Instrumental conditioning of sensorimotor cortex EEG spindles in the waking cat. Physiology \& Behavior, 3(5), 703-707. 